\title{
Neuroevolution of Controllers for Self-Organizing Mobile Ad Hoc Networks
}

\author{
David B. Knoester and Philip K. McKinley \\ Department of Computer Science and Engineering \\ Michigan State University \\ East Lansing, Michigan 48824 \\ Email: $\{d k$, mckinley $\} @$ cse.msu.edu
}

\begin{abstract}
This paper describes a study in the use of neuroevolution to discover controllers for a simulated mobile ad hoc network. Neuroevolution is a technique whereby an evolutionary algorithm is used to produce artificial neural networks that solve a user-defined task. Here, we use neuroevolution to study a generic coverage-based problem, where agents in the network are to maximize the area covered by the largest connected component of the network. An example application for this work is the discovery of control algorithms for an ocean-monitoring mobile network. While this is a challenging problem domain for neuroevolution, results of our experiments reveal three important characteristics to be considered when using such an approach. Specifically, we found that approaches that implicitly reduce entropy, while explicitly addressing self-organization and scalability, are capable of discovering behaviors that remain stable even when they control networks of different sizes than were evaluated during evolution. This result suggests that neuroevolution may be a viable strategy for discovering controllers for self-organizing multi-agent systems.
\end{abstract}

Keywords-Evolutionary algorithm, neuroevolution, selforganization, mobile ad hoc network.

\section{INTRODUCTION}

From large-scale permanent networks such as the Internet to smaller temporary networks such as mobile ad hoc wireless networks (MANETs), network creation and topology maintenance algorithms are a fundamental aspect of distributed computing systems. In fixed networks, topology maintenance is typically performed by routers, while in overlay networks, a logical communication network is created and maintained by applicationlevel software [1]. In MANETs, the multitude of routing protocols available, and their varying tradeoffs related to reliability and overhead, complicate the engineering of reliable mobile systems [2]. Methods that are able to adapt to the environment in which they are deployed, especially in those systems that interact with physical systems, are needed [3].

Many approaches to solving complex problems in distributed computing have been inspired by obser- vations of biological systems. For example, Dorigo et al. [4] and Baldasarre, Parisi, and Nolfi [5] have studied coordinated behavior of robotic swarms where individuals were able to physically attach to one another, much like army ants, and the slime mold Physarum polycephalum has been shown to form networks that are as efficient and fault-tolerant as real-world transportation networks [6]. Moreover, certain natural behaviors have been codified as generic patterns for use in a wide variety of applications [7]. Yet, determining how these bio-inspired methods can be used to engineer scalable, reliable, and decentralized systems, remains a challenging task.

In this study, we use neuroevolution to discover controllers for a simulated network of mobile autonomous communicating agents, similar to a MANET. Neuroevolution is a technique whereby an evolutionary algorithm (EA) is used to produce artificial neural networks (ANNs). Specifically, here we use NEAT [8] to solve a coverage problem, where nodes in a MANET are required to distribute themselves on a grid while maintaining network connectivity. The ANNs produced by NEAT were used as controllers for both the movement and communication behavior of nodes in the network, and all nodes in a given network executed a copy of the same evolved ANN. Nodes were provided with simulated radios, and were able to broadcast to their neighbors within a limited range. Whether agents were able to communicate with each other was defined by whether a neighbor was transmitting and also by distance between individuals.

The main contribution of this work is to demonstrate a systematic, evolution-based method by which such coverage problems can be addressed, and to show how stability, self-organization, and scalability can be integrated into fitness functions that produce effective solutions for such problems. These results hint at an evolutionary algorithm-based approach by which largescale multi-agent control can be achieved. 


\section{RELATED WORK}

Traditionally a subfield of distributed artificial intelligence, distributed problem solving is the use of multiple, semi-autonomous, and cooperating agents to solve a problem [9]. Occasionally, a distinction is drawn between decomposition/distribution approaches, and those that rely on autonomous interactions among agents, known as multi-agent systems (MAS) [10]. The task of controlling the formation of a group of agents has been examined from different perspectives, including graphstability, switching hierarchical control strategies, adaptive gradient climbing, and others [11]. Here, instead of engineering control algorithms for the movement of agents, we employ neuroevolution to automatically discover controllers for a mobile network.

In the broader context of MAS, the taxonomy provided by Panait and Luke [10] places our study in the team learning category, where a single evolving individual encodes the behavior for the entire team (mobile network, in this case). Moreover, the agents in this study engage in direct communication through messagepassing, and no mechanism for indirect communication (e.g., stigmergy) is provided. Finally, the scenario that we have selected for study is related to cooperative target observation, where a group of agents is tasked with collective observation of a target. The specifics of our scenario will be explained in more detail in Section III.

Within evolutionary computation, numerous techniques have been proposed to evolve cooperative teams that solve tasks. Waibel, Keller, and Floreano survey the work done in this area [12]. Their classification highlights whether the group is homogeneous or heterogeneous and whether selection is performed at the level of the individual or team. Additionally, they compare the performance of four types of teams: (1) homogeneous teams using individual selection; (2) homogeneous teams using group selection; (3) heterogeneous teams using individual selection, and (4) heterogeneous teams using group selection, on four cooperative tasks. Their results indicate that the genetic composition of groups and level of selection are key factors in evolving groups that perform cooperative tasks, and in general, find that homogeneous teams, as used here, tend to outperform other approaches.

Neuroevolution is a kind of evolutionary algorithm that evolves ANNs. NEAT (NeuroEvolution of Augmenting Topologies) [8], described in more detail in Section III, has previously been used to evolve teams of agents through real-time interaction with a player, where each agent was controlled by an instance of an evolved neural network [13]. Neuroevolution has also produced adaptive teams, where agents with identical neural networks nonetheless exhibit different behaviors [14]. Our study leverages NEAT to address problems in the control of MANETs.

Quintão, Nakamura, and Mateus [15] have studied dynamic coverage problems, and used an evolutionary algorithm to optimize the coverage of sensors set in fixed locations. Hauert, Zufferey, and Floreano [16] have also studied the use of a neural network controller for unmanned aerial vehicles (UAVs), where the ANN was evolved via a genetic algorithm. Here, the UAVs were to establish and maintain a multi-hop communications link between a base station and a target without global or agent-relative positioning information. Finally, D'Ambrosio et al. [17] investigated the use of derivatives of NEAT on a coverage-based problem, where agents were able to sense the boundaries of their environment, but were not able to sense other agents. In the study presented here, we investigate the control of agents that are able to sense their positions in an open environment, but yet must coordinate to solve a coverage-related problem.

In a previous study, we used a variety of different neuroevolutionary approaches to discover neural network controllers for nodes in a MANET [18]. The main result of our prior study was that, regardless of the specific neuroevolutionary approach, the difficulty of controlling the network increased with the number of nodes. In the study presented here, we again use neuroevolution to discover controllers for a MANET. However, here we focus on one neuroevolutionary system, NEAT, and propose solutions to three different aspects of this problem that were previously found to be challenging: network stability, self-organization, and scalability.

\section{METHODS}

\section{A. Grid-Coverage Problem}

Figure 1 depicts a mobile network for oceanic monitoring. In this scenario, agents in the network must not only coordinate their movement in order to remain connected via wireless links, but must also spread out to maximize the coverage area of their sensors. As a first step toward evolving solutions to such problems, we have defined the grid-coverage problem, where a mobile network of autonomous agents is to maneuver into a grid-like formation within a virtual physical environment, while maintaining network connectivity.

From an operational perspective, solutions to the gridcoverage problem could be used to provide high-level movement commands (a "scaffold") to MANETs in a 


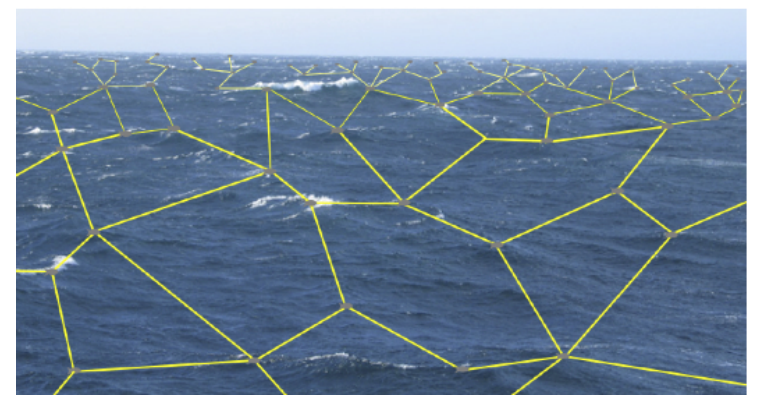

Figure 1. Illustration of a network of mobile aquatic sensors for oceanic monitoring. Such a network could be used for monitoring oil spills and studies of ocean life.

variety of different settings, including oceanic monitoring, airborne observation, and ground-based target observation. In this study, we focus on a scenario where the MANET is to navigate from an initial configuration to a fixed position within a given amount of time. Ideally, this fixed position would maximize sensor coverage. Once the network stabilizes, additional tasks (such as data gathering or target observation) could be performed, though we do not address such applications here.

Figure 2(a) depicts the initial configuration for a network whose behavior will be evolved via neuroevolution. As shown, a $6 \times 6$ grid of cells, each $4 \mathrm{~m} \times 4 \mathrm{~m}$ in size, is arrayed in a flat environment. Each of 16 mobile agents is modeled as rolling sphere. The physicsbased characteristics of this environment are calculated by the Open Dynamics Engine (ODE, version 0.11.1, http://www.ode.org). Within this environment, agents have a variety of sensors and effectors, summarized in Table I. Figure 2(b) depicts this same network after 4s of control by an evolved neural network. Agents that are within $10 \mathrm{~m}$ of each other are able to communicate, which are shown by links among agents. When an agent transmits (by raising its tx effector above 0 ), the appropriate rx sensor of each agent within $10 \mathrm{~m}$ is set to the inverse of the distance from sender to receiver. The particular rx sensor used for a given transmission is based on the orientation of the agents in space; each rx sensor covers a $90^{\circ}$ "pie slice" around the agent. When multiple transmissions are received, the strongest signal (nearest agent) is used.

Evaluating the performance $G(M, n)$ of a MANET on the grid-coverage problem is a straightforward process of measuring the fraction of cells that are occupied by nodes. Specifically:

$$
G(M, n)=\frac{\operatorname{cells}\left(M_{c}\right)}{\min \left(n,\left|M_{c}\right|\right)}
$$

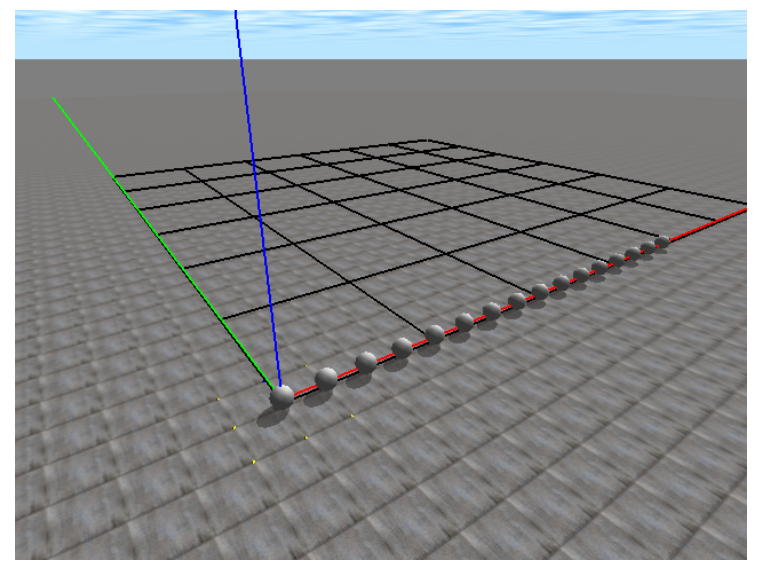

(a)

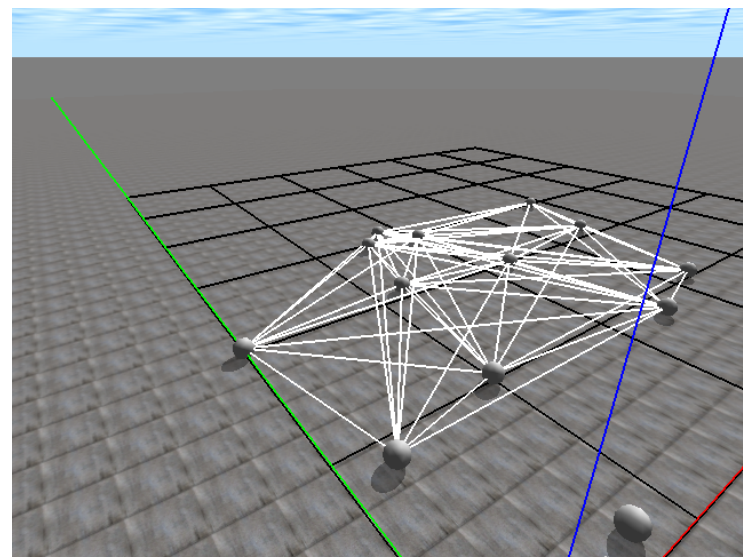

(b)

Figure 2. Starting configuration of agents (a) and position of agents $4 \mathrm{~s}$ into a simulation (b). White lines represent connections between agents; agents can transmit data only to those other agents to which they are connected. The grid agents are to cover is outlined with black lines; $x, y$, and $z$ axis represented by red, green, and blue lines, respectively.

where $M$ is the MANET being evaluated, $M_{c}$ is the largest connected component of $M$ based on the communication network, cells $\left(M_{c}\right)$ calculates the number of unique cells in the grid that are occupied by at least one agent in $M_{c}$, and $n$ is the number of cells present in the grid. In all of the studies presented here, $n=36$ (the grid is always $6 \times 6$ ).

\section{B. NEAT Overview}

NEAT [8] is fundamentally a genetic algorithm that uses a direct encoding, where each gene encodes either a neuron or link in an artificial neural network. A key advantage of NEAT compared to other neuroevolutionary approaches is that it is able to evolve the structure of the neural network. Specifically, NEAT starts with ANNs that include neither a hidden layer nor 
Table I

SENSORS AND EFFECTORS OF INDIVIDUAL AGENTS FOR THE GRID-COVERAGE PROBLEM.

\begin{tabular}{|c|c|c|}
\hline Name & Type & Description \\
\hline$r \times 0 \ldots r \times 3$ & sensor & $\begin{array}{l}\text { directional radio receiver; } \\
\text { each sensor covers a } 90^{\circ} \\
\text { "pie slice" around the } \\
\text { agent }\end{array}$ \\
\hline v0...v2 & sensor & velocity vector $(x, y, z)$ \\
\hline p0...p2 & sensor & absolute position $(x, y, z)$ \\
\hline f0...f2 & effector & force vector $(x, y, z)$ \\
\hline tx & effector & $\begin{array}{l}\text { broadcasts a message to all } \\
\text { agents within range; appro- } \\
\text { priate rx sensor is always } \\
\text { set to the inverse of the dis- } \\
\text { tance between sender and } \\
\text { receiver }\end{array}$ \\
\hline
\end{tabular}

recurrent connections. Over time, using a process called complexification, NEAT is able to evolve genomes that include both hidden neurons and recurrence. Thus, NEAT does not bias or constrain evolution to any particular network topology. In this study, NEAT is used for all experiments, with relevant configuration options summarized in Table II.

Table II

RELEVANT CONFIGURATION PARAMETERS FOR NEAT; THOSE NOT LISTED ARE SET TO THEIR DEFAULT VALUE.

\begin{tabular}{ll}
\hline \hline Parameter & Value \\
\hline PopulationSize & 100 \\
MaxGenerations & 200 \\
MutateAddNodeProbability & 0.03 \\
MutateAddLinkProbability & 0.05 \\
MutateDemolishLinkProbability & 0.03 \\
MutateLinkWeightsProbability & 0.8 \\
MutateOnlyProbability & 0.25 \\
MutateLinkProbability & 0.1 \\
AllowAddNodeToRecurrentConnection & 1.0 \\
AllowRecurrentConnections & 0.0 \\
AllowSelfRecurrentConnections & 0.0 \\
OnlySigmoidHiddenNodes & 1.0 \\
\hline
\end{tabular}

\section{EXPERIMENTS AND RESULTS}

\section{A. Motivating Experiment}

Our first experiment investigated the evolution of neural networks that could solve the grid-coverage problem, and it provides the motivation for the following experiments. Specifically, the fitness function used here was simply that shown in Equation 1, which was evaluated after 10 s of simulation time.

Figure 3 plots the mean normalized fitness of the dominant (most fit) individual per generation across 30 separate trials for both 8-node $(F F 8)$ and 16node ( $F F 16)$ networks. The (very small) error bars in this and other figures are $95 \%$ confidence intervals constructed via 200-fold bootstrapping. We define normalized fitness as $f / f_{\max }$, where $f_{\max }$ is the maximum possible fitness achievable given the fitness function and network size. In this treatment, fitness and normalized fitness are identical, although that is not the case in many of the following experiments. In Figure 3, we see that the dominant individuals in 8-node networks reach their optimal fitness after approximately 40 generations, while 16 -node networks averaged only $71.5 \pm 0.6 \%$ after 200 generations.

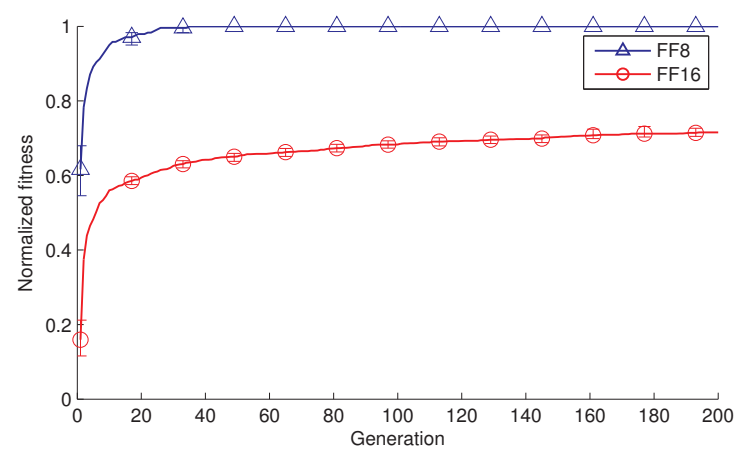

Figure 3. Normalized fitness of 8- and 16-node fixed-size networks using a fitness function that rewards only for grid-coverage.

Upon examination of the evolved behaviors, the reason for the decline in fitness of 16-node networks became apparent: For both 8- and 16-node networks, the evolved strategies were primarily stochastic, and relied upon the continual movement of nodes throughout the environment. One such behavior is depicted in Figure 4, which shows the position and velocity vectors of each node in the 16-node network over 20 s of simulation time. In this figure, nodes start in an array along the $x$-axis, and generally move in a spiral pattern within the region bounded by the grid. A movie of this behavior (and others described in the following sections) is available at: http://www.cse.msu.edu/thinktank/manet. This behavior was also prevalent in the smaller 8-node networks. The reason this strategy is effective is related to the size of the grid relative to the number of nodes in the network. On average, if the grid is large relative to the size of the network, and nodes maintain some distance between each other while moving over the 
grid, nodes are likely to be in different cells at the time of fitness evaluation. This strategy is less effective with larger MANETs because nodes are more likely to occupy the same cell. Interestingly, this strategy is not unlike that exhibited in flocks of starlings [19], where individuals adjust their movements to maintain a "safe" distance amongst themselves.

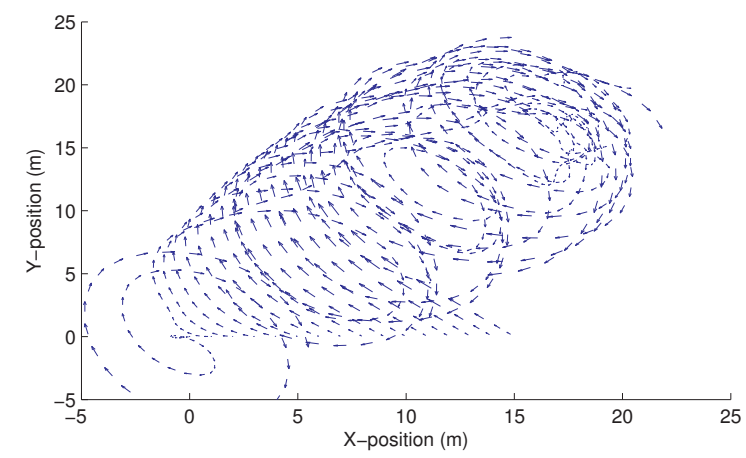

Figure 4. Sample velocity and position of a 16-node network following 20s of control (FF16 treatment). Nodes start at $(x, 0)$, and then move in a spiral for the remainder of the simulation.

However, from the perspective of engineering controllers for a MANET, these evolved behaviors are lacking in two significant ways: First, the evolved behaviors are unstable. We expected to see behaviors where nodes would deterministically move to unique cells in the grid and stop moving. Instead, the nodes moved continually in a spiral pattern, which would waste energy in a real network. Second, these behaviors do not scale. We hoped to evolve effective behaviors for networks of varying size. However, we found that normalized fitness decreased as network size increased, a result which we verified on larger 32-node networks and both larger and smaller grids. In all cases, the key factor was the number of nodes being controlled, which is known to be a challenge for multi-agent systems [10]. In the following experiments, we examine ways to improve network stability and scalability.

\section{B. Stable Networks}

Based on the behaviors observed in the previous experiment, we first explored ways to improve network stability. The goal here was to discover networks whose nodes would move to and then settle in a location, instead of continuing to move throughout the environment. Our first approach was to devise a fitness function that included a speed component, such that networks with nodes that were moving slowly at the time of fitness evaluation would have a higher fitness than a network whose nodes were moving quickly. This fitness function, $S F(M)$, rewarded for a reduction in the average speed at which nodes in $M$ (the MANET) were moving, specifically:

$$
S F(M)=\frac{G(M, 36)}{10 \cdot \max (0.1, \overline{\operatorname{spd}(M)})}
$$

where $G(M, 36)$ is performance on the 36-cell gridcoverage problem, and $\overline{\operatorname{spd}(M)}$ is the average speed of all nodes in $M$ at the end of the simulation. The other components of this fitness function, scaling by 10 and $\max (\cdot)$, were used to ensure that the resulting fitness score is in the range [0..1]. This fitness function thus rewards MANETs that quickly move to, and settle, in a stable configuration.

Figure 5 plots the mean normalized fitness of the dominant individual per generation over 30 trials on networks of 16 nodes. Surprisingly, the mean normalized fitness of dominant individuals under this treatment is $55.3 \pm 1.2 \%$ after 200 generations, which is even worse than in the base FF16 case. Upon examination of the evolved behaviors, however, we did indeed find that the networks exhibited the desired behavior, where nodes stabilized into a fixed position after reaching their target cells, although many nodes shared occupancy of cells.

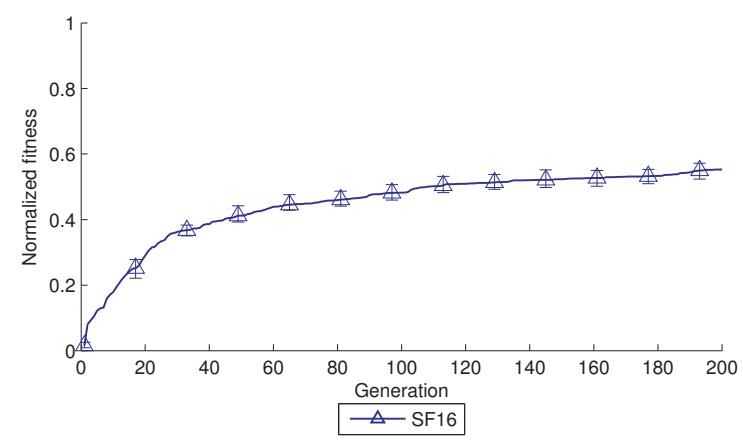

Figure 5. Normalized fitness for 16-node networks that are rewarded for reduced speed.

\section{Reducing Entropy}

One key difference we noticed between this behavior and that shown in Figure 4 is that here the behavior of individual nodes was based on their starting location in the environment, and did not appear to be stochastic. Information entropy is frequently used to characterize stochastic processes [20]. To measure information entropy, $H(X)$, of a mobile network, we leverage the following definition:

$$
H(X)=-\sum_{i=1}^{n} p\left(x_{i}\right) \log _{2} p\left(x_{i}\right)
$$


where $X=\left\{x_{1}, \ldots, x_{n}\right\}$ is the discrete random variable representing the states of the network, and $p(\cdot)$ is the probability mass function of $X$. We define the states of $X$ based on the cells of the grid occupied by nodes in the network. Specifically, each possible value of $X$ is a length- $k$ bitstring, where $k$ is the number of cells in the grid (36, in this study). A " 1 " at position $i$ in this bitstring represents that cell $i$ is occupied by at least one node, while a " 0 " indicates that cell $i$ is not occupied. The frequencies of these states during a simulation defines $p(\cdot)$. Intuitively, a high value for information entropy represents a network that exhibits many states with low frequency, while a low value represents a network that exhibits few states with high frequency. We assume that low information entropy is a desirable property of engineered systems. For brevity, in the remainder of this paper we will refer to this calculation as the entropy of a network.

Figure 6 plots the mean entropy for the dominant individuals per generation over all 30 trials for two treatments: FF16, described in Section IV-A, whose fitness function did not include a velocity component; and $S F 16$, described in this section, where a reward for reduced velocity was included as part of fitness. As would be expected, here we see that entropy of the $S F 16$ treatment is significantly lower than that of the FF16 treatment, indicating a more stable behaviors.

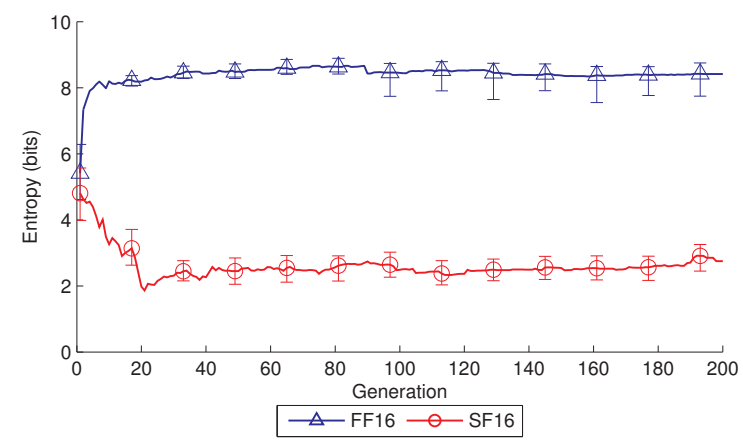

Figure 6. Entropy on 16-node networks under a treatment rewarding only for grid-coverage $(F F 16)$ and the grid-coverage plus reduced velocity $(S F 16)$.

To further explore the relationship between entropy and behavior, we also devised a fitness function that rewarded explicitly for a reduction in entropy, with no consideration given to speed. Though their performance on the grid-coverage problem was similar, the evolved behaviors were markedly different. Specifically, when using a fitness function that rewarded for reduced entropy instead of average speed, nodes were likely to start by moving very slowly, but then would accelerate as the simulation progressed, eventually moving off the grid (data not shown). For this reason, our subsequent experiments include speed-reduction, which implicitly reduces entropy, as a component of fitness.

\section{Reactive Networks}

Thus far, we have considered several approaches to the evolution of controllers and identified three main challenges. The first, which we have addressed by rewarding for a reduction in average node speed, was that evolved behaviors tended to be stochastic. Second, we found that when fitness includes a reward for stability, the evolved behaviors were not reactive. Finally, we found that fitness decreased as the number of nodes in the network increased, identifying scalability as a concern as well. To address these final two challenges, we investigated two new approaches that varied the number of nodes in the network during the course of the simulation. Our hypothesis was that if the number of nodes in the simulation changed over time, then individual nodes would have to react to each other in order to achieve a high fitness, and that this would result in reactive and scalable behaviors.

For these two approaches, we initialized each simulation with a MANET comprising only 2 nodes. In the first treatment, $S V 2$, we unconditionally added 2 nodes to the simulation environment every $5 \mathrm{~s}$, and ran the simulation for a total of 120 s ( 48 total nodes at the end of the simulation; the grid remained unchanged at 36 cells), at which point fitness was calculated. The second treatment, $S V 2 G$, was configured identically, except that nodes were added only if the existing network had a normalized fitness greater than $80 \%$. This second treatment was inspired by the idea of "building blocks" in genetic algorithms. In this case, the building block is a behavior for a smaller network. In both cases, we used the fitness function shown in Equation 2. We note that for these treatments, the calculation of normalized fitness is done with respect to the number of nodes that would be present in the network assuming optimal control.

Figure 7 plots the mean normalized fitness of the dominant individual per generation over 30 trials of each of these two treatments. In general, fitnesses and evolved behaviors under these two treatments were poor. In the $S V 2$ treatment, where nodes were unconditionally added, a common behavior was for nodes to slowly move across the grid, relying on the addition of new (also slow-moving) nodes to achieve higher fitness.

The poor performance of the $S V 2 G$ treatment is explained by the small number of nodes that were being controlled: In the best case overall, only 12 nodes 


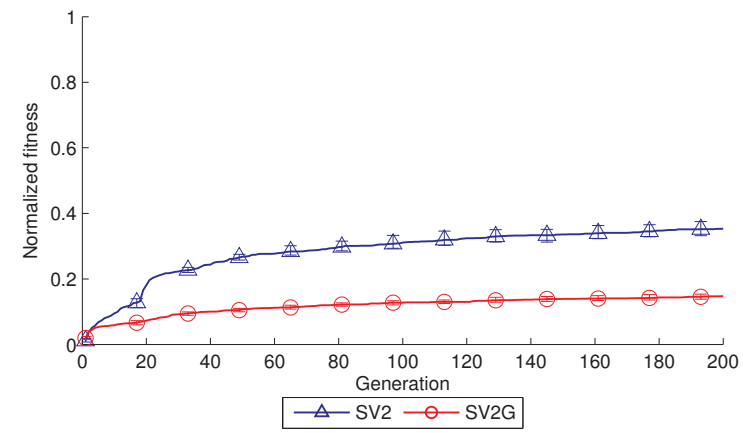

Figure 7. Normalized fitness for treatments where nodes are added to the simulation unconditionally ( $S V 2)$, and conditionally based on the existing network reaching $80 \%$ of its possible fitness $(S V 2 G)$.

(out of a possible 48) were controlled following 200 generations of evolution. However, we did notice that the more successful behaviors appeared to depend on interaction among individuals, which we tested by turning communication off and re-evaluating the dominant evolved solutions.

This property, where individuals react to each other locally without regard to a global pattern, is an example of self-organization. There are numerous definitions for self-organization. For example, Haken [21] states that, "A system is self-organizing if it acquires a spatial, temporal, or functional structure without specific interference from the outside." Another definition is provided by Camazine [22]: "Self-organization is a process in which pattern at the global level of a system emerges solely from numerous interactions among the lowerlevel components of the system." Likewise, numerous metrics have been proposed for how to measure selforganization [23]-[25]. However, in the context of evolving a solution to the grid-coverage problem, what is needed is a way to ensure that the neural networks produced via evolution are communicating to solve the overall problem. We thus define operational selforganization, $S_{o p}(x)$, of a neural network $x$, as:

$$
S_{o p}(x)=\frac{f(x)-f_{n c}(x)}{f(x)+f_{n c}(x)}
$$

where $f(x)$ is the fitness of the neural network $x$ with communication among agents enabled and $f_{n c}(x)$ is fitness with communication among agents disabled. $S_{o p}(x)$ has the interesting property that values greater than zero indicate the presence of self-organization (communication is beneficial), while values less than or equal to zero indicate the lack of self-organization (communication is harmful or neutral).

We calculated operational self-organization for the dominant solutions from all of the treatments previously presented; results are shown in Figure 8. Surprisingly, we see that the original fast-moving, highly entropic treatment $(F F 16)$ also had the highest degree of operational self-organization. Also surprising is that the $S F 16$ treatment, which rewarded for reduced velocity and had only 3 bits of entropy, exhibited the least selforganization. This result, where stable solutions had the least self-organization, led us to include operational selforganization as a component of fitness, as will be seen shortly. First, however, let us consider scalability.

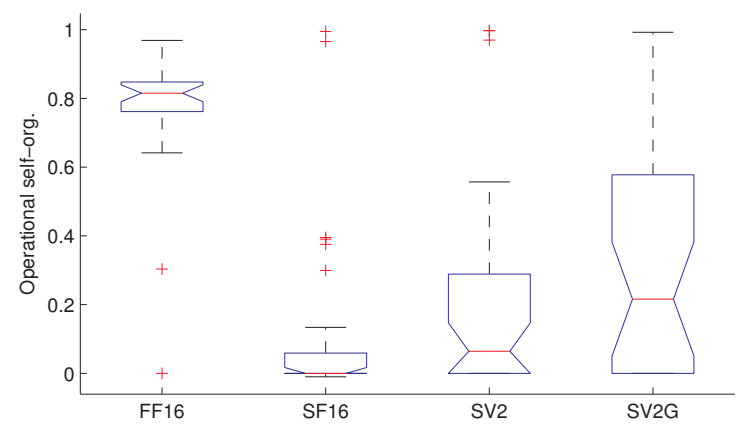

Figure 8. Operational self-organization of various treatments. Notably, the treatment with the greatest entropy $(F F 16)$ also exhibited the most self-organizatin.

\section{E. Scalable Networks}

Scalability remains a key challenge not only for multi-agent systems [10], but also for many types of distributed systems. Ideally, we wish to discover controllers that scale to large numbers of nodes and can gracefully accommodate node churn. While the tendency for a neural network controller to exhibit scalable behavior can be difficult to capture analytically, we can measure it operationally, in a manner similar to the measure for operational self-organization presented above.

Figure 9 depicts performance of the dominant individuals from the $S V 2 G$ treatment on networks that vary in size from 1 to 30 nodes (30 dominant individuals were each tested on 30 different network sizes). Here we see very high (near-optimal) fitness for small networks, but performance quickly falls off as the number of nodes is increased. In general, we see that performance declines as additional nodes are available for control, and that the $S V 2 G$ treatment performs well when controlling small networks of 6 or fewer nodes. We also analyzed the $F F 16$ treatment, and the results were similar, although fitness declined more slowly as network size increased (data not shown).

Based on the results in Figure 9, we devised a heuristic for measuring approximate scalability, $C(x)$, 


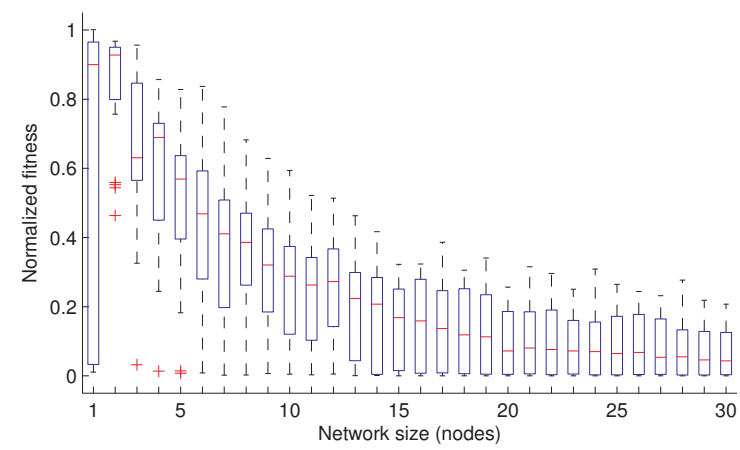

Figure 9. Normalized fitness vs. network size for the dominant individuals from the $S V 2 G$ treatment

of neural networks that control the nodes of a MANET. Effectively, $C(x)$ is the area under the curve shown in Figure 9, scaled to [0..1]. Formally, we define the approximate scalability, $C(x)$, of a neural network $x$ as:

$$
C(x)=\frac{\operatorname{trapz}_{i=1}^{|S|} f\left(x, s_{i}\right)}{|S|}
$$

where $S=\left\{s_{1}, \ldots, s_{n}\right\}$ is a set of network sizes over which $x$ is evaluated, $f\left(x, s_{i}\right)$ is the normalized fitness achieved by a network of size $s_{i}$ where each node is controlled by neural network $x$, and trapz $(\cdot)$ calculates the trapezoidal area $(\operatorname{trapz}(\cdot)$ is an approximation of the definite integral). We note that this definition of scalability is dependent on the set of network sizes $(S)$ being evaluated, but for sufficient $S, C(x)$ will approach 1.0 for scalable neural network controllers.

Figure 10 plots the scalability of the final dominants of the four treatments previously described. As with self-organization, here we again see that the $F F 16$ treatment produced the most scalable solutions, although the relative ordering of the remaining treatments is different here than it was for self-organization.

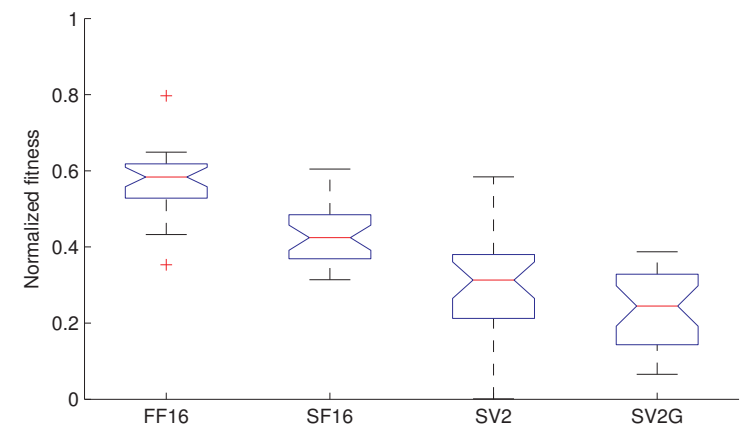

Figure 10. Scalability of four treatments, where treatment $F F 16$, which also exhibited the most entropy and self-organization, is more scalable than other approaches.
We next analyzed correlation among the different values of entropy, self-organization, and scalability that were measured from the dominant individuals in each of these four treatments. First, we found no statistically significant correlation between entropy and self-organization (Spearman rank correlation, $\rho=$ $-0.007, p=0.968)$, which indicated that they are independent characteristics, and can thus be included as separate components of fitness functions. Second, we found a negative correlation between entropy and scalability $(\rho=-0.454, p=0.011)$, which reinforced the idea that a reduction in entropy is a desirable property in our search for scalable behaviors (as entropy decreased, scalability increased). Finally, we found a positive correlation between self-organization and scalability ( $\rho=0.376, p=0.040)$, which indicates that selforganization may be an important "building block" for scalable behaviors. Therefore, in the next experiment, we investigate an approach intended to reduce entropy while simultaneously increasing self-organization and scalability.

\section{F. Stable, Self-organizing, and Scalable Networks}

In this section we present our final experiment and introduce a method for evolving controllers that produces stable, self-organizing, and scalable behaviors. Similar to previous treatments, the fitness function presented here includes a component for reducing average speed (which has the side-effect of reducing entropy), as well as components for increasing self-organization and scalability. Specifically, we define the fitness function, $E S S(x)$, where $x$ is the neural network being evaluated, as:

$$
\operatorname{ESS}(x)= \begin{cases}S F(x)+ & \\ \max \left(0, S_{o p}(x)\right. & \text { if } S_{o p}(x) \leq 0 \\ S F(x)+ & \\ \max \left(0, S_{o p}(x)+\right. & \\ C(x) & \text { if } S_{o p}(M)>0\end{cases}
$$

where $S F(x)$ is performance on the grid-coverage problem, as defined earlier in Equation 1; $S_{o p}(x)$ is selforganization, as defined in Equation 4; and $C(M)$ is the measured scalability of $x$ tested on networks of size 8, 16, 24, and 32, as described in Equation 5.

Evaluating the fitness of a neural network $x$ using Equation 6 proceeds as follows. First, performance of a network comprising 8-nodes controlled by $x$ is evaluated on the grid-coverage problem using Equation 1. Then, performance is re-evaluated in a simulation in which communication is disabled, allowing us to calculate operational self-organization using Equation 4. If the resulting value for $S_{o p}$ is positive, we then evaluate 
$x$ on networks of size 16,24 , and 32 . The normalized fitness values for all 4 different network sizes are then used in Equation 5 to calculate the approximate scalability of $x$.

Figure 11 depicts mean normalized fitness, entropy, operational self-organization, and scalability for the dominant individuals from 30 trials of the ESS treatment. This treatment was allotted 3,000 generations primarily because fitness was still improving after 200 generations. Here we see consistent low entropy and high operational self-organization, while scalability slowly improves. We note that the slow improvement in fitness is primarily driven by scalability.

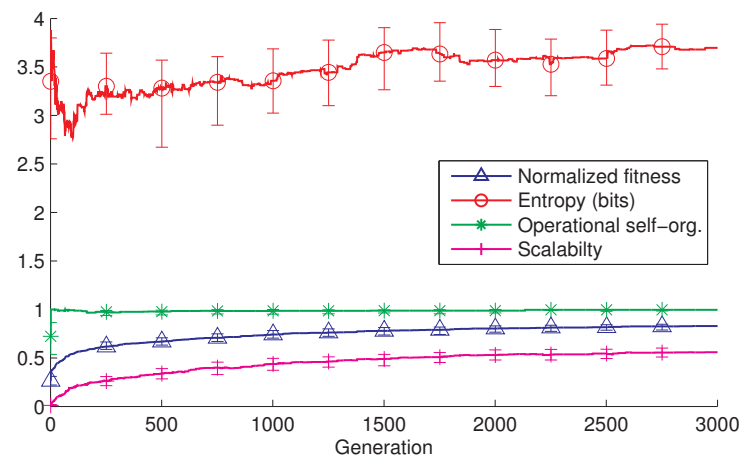

Figure 11. Characteristics of the dominant individuals evolved under treatment $E S S$.

Interestingly, we observed that rewarding explicitly for scalability had an immediate effect, even after only 200 generations. Figure 12 plots normalized fitness vs. network size after 200 and after 3,000 generations. Compared to Figure 9, here we see more consistent performance across network sizes, and a less pronounced decline in fitness.

Figure 13 depicts the position and velocity of a 16node network during 20 s of simulation for one of the dominant individuals evolved here. This figure shows behavior both when communication is disabled (red), and enabled (blue). When nodes are unable to communicate, as during the latter half of the calculation of operational self-organization, nodes begin by slowly moving away from the grid, and their behavior does not change. When nodes are able to communicate, their first movements are again away from the grid, however a subset rapidly advances across the grid, reversing direction just short of the grid's boundary. The remaining nodes then also advance, somewhat more slowly, stopping near the middle of the grid. Nodes are thus actively using the presence of neighbors to alter their behavior, a characteristic of self-organization.

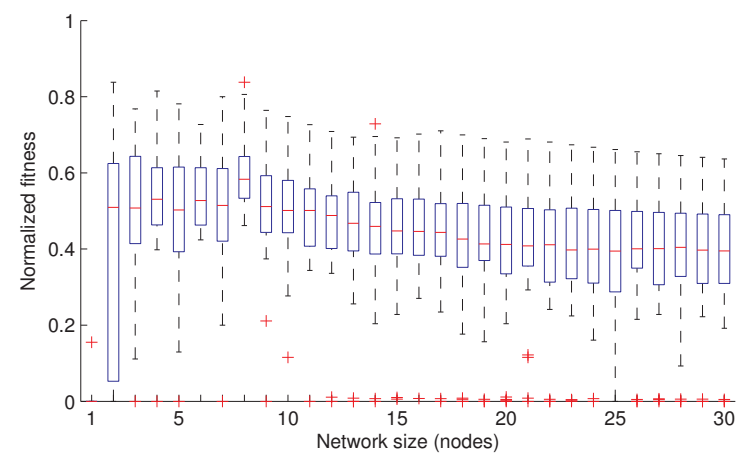

(a)

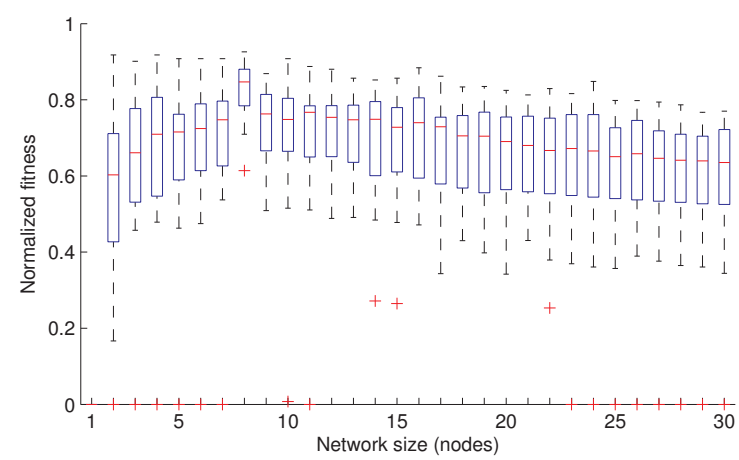

(b)

Figure 12. Normalized fitness vs. network size for the dominant individuals from after 200 (a) and 3,000 (b) generations under the ESS treatment.

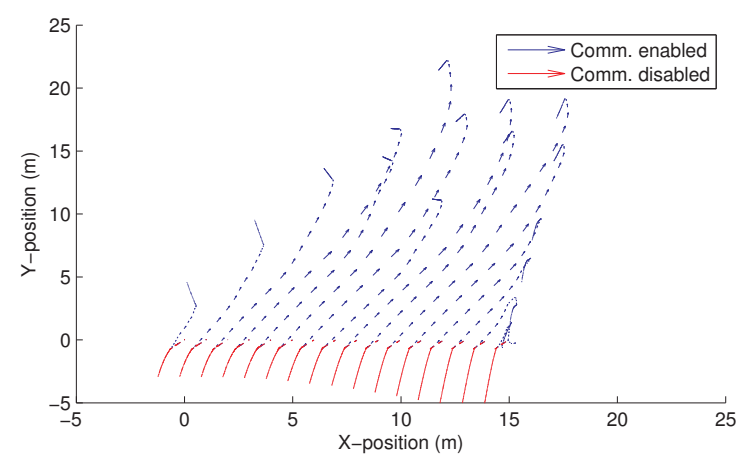

Figure 13. Velocity and positions of an example individual evolved under the ESS treatment with communication enabled and disabled.

\section{CONCLUSION}

Experiments described herein indicate that neuroevolutionary approaches that reduce entropy, while increasing self-organization and scalability, are capable of discovering effective controllers for mobile ad hoc networks. Moreover, we found that the evolved behaviors remained stable even when controlling different numbers of nodes than they had originally been evaluated 
with. This result suggests an approach by which control of large-scale multi-agent systems might be achieved. There are, of course, many different ways that these factors could be integrated into evolutionary algorithms. In future work, we plan to investigate multiobjective optimization approaches for these characteristics, with a goal of achieving reliable, self-organizing, large-scale systems.

\section{FURTHER INFORMATION AND ACKNOWLEDGMENTS}

Animations of agent behavior can be found at http:// www.cse.msu.edu/thinktank/manet. This work was supported in part by National Science Foundation grants DBI-0939454, CNS-0915855, CCF-0820220, and CNS0751155, and by U.S. Army Grant W911NF-08-1-0495.

\section{REFERENCES}

[1] M. Jelasity and O. Babaoglu, "T-Man: Gossip-based overlay topology management," in Proceedings of the Workshop on Engineering Self-Organising Applications (ESOA), 2005, pp. 1-15.

[2] J. Broch, D. A. Maltz, D. B. Johnson, Y.-C. Hu, and J. Jetcheva, "A performance comparison of multi-hop wireless ad hoc network routing protocols," in MobiCom '98: Proceedings of the 4th annual ACM/IEEE international conference on Mobile computing and networking, 1998, pp. 85-97.

[3] W. Wolf, "Cyber-physical systems," IEEE Computer, vol. 42, no. 3, 2009.

[4] M. Dorigo, V. Trianni, E. Şahin, R. Groß, T. H. Labella, G. Baldassarre, S. Nolfi, J.-L. Deneubourg, F. Mondada, D. Floreano, and L. M. Gambardella, "Evolving self-organizing behaviors for a swarm-bot," Autonomous Robots, vol. 17, no. 2, pp. 223-245, 2004.

[5] G. Baldassarre, D. Parisi, and S. Nolfi, "Coordination and behavior integration in cooperating simulated robots," in Proceedings of the Conference on Simulation of Adaptive Behavior, 2004, pp. 385-394.

[6] A. Tero, S. Takagi, T. Saigusa, K. Ito, D. P. Bebber, M. D. Fricker, K. Yumiki, R. Kobayashi, and T. Nakagaki, "Rules for Biologically Inspired Adaptive Network Design," Science, vol. 327, no. 5964, pp. 439-442, 2010.

[7] O. Babaoglu, G. Canright, A. Deutsch, G. A. D. Caro, F. Ducatelle, L. M. Gambardella, N. Ganguly, M. Jelasity, R. Montemanni, A. Montresor, and T. Urnes, "Design patterns from biology for distributed computing," ACM Transactions on Autonomous and Adaptive Systems, vol. 1, no. 1, pp. 26-66, 2006.

[8] K. O. Stanley and R. Miikkulainen, "Evolving neural networks through augmenting topologies," Evolutionary Computation, vol. 10, no. 2, 2002.

[9] G. Weiss, Ed., Multiagent Systems: A Modern Approach to Distributed Artificial Intelligence. MIT Press, 1999.

[10] L. Panait and S. Luke, "Cooperative multi-agent learning: The state of the art," Autonomous Agents and MultiAgent Systems, vol. 11, no. 3, pp. 648-660, 2005.
[11] C. Cassandras and W. Li, "Sensor networks and cooperative control," European Journal of Control, vol. 11, no. 4-5, 2005.

[12] M. Waibel, L. Keller, and D. Floreano, "Genetic Team Composition and Level of Selection in the Evolution of Cooperation," IEEE Transactions on Evolutionary Computation, vol. 13, no. 3, pp. 648-660, 2009.

[13] K. Stanley, B. Bryant, and R. Miikkulainen, "Realtime neuroevolution in the NERO video game," IEEE Transactions on Evolutionary Computation, vol. 9, no. 6, 2005.

[14] B. D. Bryant and R. Miikkulainen, "Neuroevolution for adaptive teams," in Proceedings of the IEEE Congress on Evolutionary Computation (CEC), vol. 3, 2003.

[15] F. Quintão, F. Nakamura, and G. Mateus, "Evolutionary algorithm for the dynamic coverage problem applied to wireless sensor networks design," in IEEE Congress on Evolutionary Computation (CEC), vol. 2, 2005, pp. 1589-1596.

[16] S. Hauert, J.-C. Zufferey, and D. Floreano, "Evolved swarming without positioning information: an application in aerial communication relay," Autonomous Robots, vol. 26, no. $1,2009$.

[17] D. B. D'Ambrosio, J. Lehman, S. Risi, and K. O. Stanley, "Evolving policy geometry for scalable multiagent learning," in Proceedings of the Conference on Autonomous Agents and Multiagent Systems (AAMAS), 2010.

[18] D. B. Knoester, H. J. Goldsby, and P. K. McKinley, "Neuroevolution of mobile ad hoc networks," in Proceedings of the Genetic and Evolutionary Computation Conference (GECCO), 2010, pp. 603-610.

[19] E. Fernandez-Juricic, S. Siller, and A. Kacelnik, "Flock density, social foraging, and scanning: an experiment with starlings," Behavioral Ecology, vol. 15, no. 3, pp. 371-379, 2004.

[20] C. E. Shannon, "A mathematical theory of communication," Bell System Technical Journal, vol. 27, pp. 379423, July 1948.

[21] H. Haken, Information and Self-Organization: A Macroscopic Approach to Complex Systems. Springer, Berlin, Heidelberg, 2006.

[22] S. Camazine, J. Deneubourg, N. Franks, J. Sneyd, G. Theraula, and E. Bonabeau, Self-organization in biological systems. Princeton University Press, 2003.

[23] D. Polani, "Measuring self-organization via observers," in Advances in Artificial Life, ser. Lecture Notes in Computer Science. Springer Berlin / Heidelberg, 2003, vol. 2801, pp. 667-675.

[24] H. Schmeck, C. Müller-Schloer, E. Çakar, M. Mnif, and U. Richter, "Adaptivity and self-organization in organic computing systems," ACM Transactions on Autonomous and Adaptive Systems, vol. 5, no. 3, September 2010.

[25] D. Yamins, "Towards a theory of "local to global" in distributed multi-agent systems (I and II)," in International Joint Conference on Autonomous Agents and Multiagent Systems (AAMAS), 2005, pp. 183-190. 